\title{
Incidence of Urogenital Neoplasms in India
}

\begin{abstract}
Objective: To study and compare the national and regional incidences and risk of developing of neoplasms of individual urogenital sites using 2012 - 2014 reports from the National Cancer Registry Programme (NCRP) data. Materials and Methods: A number of incident cases, ageadjusted rates (AARs), and cumulative risk $(0-64$ years) pertaining to urogenital neoplasms, along with the ICD-10 codes, were extracted. Data on indicators, namely number of incident cases, AARs and one in a number of persons develop cancer were summarized for both the sexes in each of the cancer registries and presented region-wise in the form of ranges. Results: The proportion of all urogenital neoplasms in comparison to all cancers was $12.51 \%$ in women and $5.93 \%$ in men. Risk of development of urogenital cancers for women was maximum (1 in 50) in the North-eastern region, followed by Rural West, South, and North. For men, the risk of developing neoplasms of urogenital sites was highest ( 1 in 250). For the neoplasms of the renal pelvis and ureter, both the incidence and risk were quite low for all genders across all the regions. Cervical neoplasms had the highest incidence $(4.91$ - 23.07) among female genital neoplasms, while prostate had the highest incidence (0.82 - 12.39) among male genital neoplasms. Conclusion: Making people aware of urogenital neoplasms and their risk factors are important for the public health awareness point of view. Centers that deal with either management of urogenital cases or/and screening of genital neoplasms could serve as the designated centers for creating such awareness.
\end{abstract}

Keywords: Age-adjusted rates, cancer registry, cumulative risk, incidence, neoplasms, urogenital

\section{Introduction}

According to the GLOBOCAN 2012, almost $21.4 \%$ of incident cancer cases across the world involved major urogenital sites (urinary bladder, kidney, cervix, uterus, ovary, prostate, and testis). In India, $20.7 \%$ of incident cancer cases involved these sites, with $17.7 \%$ cancer-related deaths attributable to them. ${ }^{[1]}$ Despite well understood and extensively described epidemiological and etiological profiles of urogenital neoplasms, Indian efforts for prevention, screening, and treatment have either been scant or misguided. ${ }^{[2,3]}$ Cervical cancer is a case in point. Cervical cancer was the second most common cancer in India, with almost $10 \%$ of all cancer-related deaths attributable to it. ${ }^{[1]}$ Despite being amenable to early detection, no national level or large-scale screening program exists. ${ }^{[3]}$ In fact, a large-scale situational analysis of public health-care centers in the National Capital Region revealed a horrific lack of basic screening, diagnostic, and treatment facilities. ${ }^{[4]}$

This is an open access journal, and articles are distributed under the terms of the Creative Commons Attribution-NonCommercial-ShareAlike 4.0 License, which allows others to remix, tweak, and build upon the work non-commercially, as long as appropriate credit is given and the new creations are licensed under the identical terms.

For reprints contact: reprints@medknow.com
General Indian populace remains largely ignorant of the existence of most urologic and genital tract cancers let alone their risk factors. ${ }^{[5,6]}$ While smoking has been widely established as a significant risk factor for the development of neoplasms of kidney, bladder, cervix, and prostate, the tobacco awareness programs continue to focus only on lung and head-and-neck cancers. Coupled with horrifyingly inadequate urologic services, this makes for a grim situation. ${ }^{[7]}$

This willful neglect continues in the area of published medical literature. While cervical cancer remains a hot topic for researchers in India, cancers of other urogenital sites remain cold spots. This study attempts to detail, summarize, and compare the national and regional incidences and risk of development of neoplasms of individual urogenital sites using 2012-2014 reports from the National Cancer Registry Programme (NRCP), with the hopes of emphasizing a neglected chunk of cancer burden, that is amenable to screening and efficient primary prevention. How to cite this article: Labani S, Rawat D,
Asthana S. Incidence of urogenital neoplasms in
India. Indian J Med Paediatr Oncol 2018;39:446-51.

\section{Satyanarayana Labani, Dishank Rawat, Smita Asthana}

Division of Epidemiology and Biostatistics, Institute of Cytology and Preventive Oncology, Indian Council of Medical Research, Noida, Uttar Pradesh, India
Address for correspondence: Dr. Satyanarayana Labani, Division of Epidemiology and Biostatistics, Institute of Cytology and Preventive Oncology, Indian Council of Medical ResearchI-7, Sector-39, Noida - 201 301, Uttar Pradesh, India.

E-mail: labani_icpo@hotmail. com

Access this article online Website: www.ijmpo.org

DOI: 10.4103/ijmpo.ijmpo_76_17 Quick Response Code:

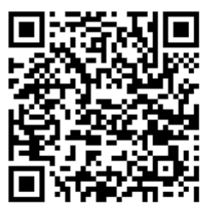




\section{Methods}

This study utilized data from published reports of 27 Indian PBCRs, compiled under NRCP by the Indian Council of Medical Research. A number of incident cases, age-adjusted rates (AARs), and cumulative risk (0-64 years) pertaining to urogenital neoplasms, along with the ICD-10 codes, were extracted. Following sites were considered as follows: vulva (C51), vagina (C52), cervix uteri (C53), corpus uteri (C54), uterus unspecified (C55), and ovary (C56); other female genital (C57), placenta (C58), penis (C60), prostrate (C61), testis (C62); and other male genital (C63), kidney (C64), renal pelvis (C65), ureter (C66), urinary bladder (C67), and unspecified urinary organs (C68). C6468 were clubbed under

Based on the location, 27 registries were divided into seven regions (years referenced mentioned in the brackets) as follows:

i. North: Delhi (2012), Patiala (2012-2014)

ii. South: Bengaluru (2012), Chennai (2012-2013), Kollam (2012-2014), and Thiruvananthapuram (2012-2014)

iii. Central: Bhopal (2012-2013)

iv. Northeast: Cachar (2012-2014), Kamrup (2012-2014), Dibrugarh (2012-2014), Manipur (2012-2014), Mizoram (2012-2014), Sikkim (2012-2014), Meghalaya (2012-2014), Tripura (2012), Nagaland (2012-2014), Naharlagun (2012-2014), and Pasighat (2012-2014)

v. West: Barshi Expanded (2012), Mumbai (2012), Ahmedabad (2012-2013), Aurangabad (2012-2014), Nagpur (2012-2013), Pune (2012-2013), and Wardha (2012-2014)

vi. Rural West: Barshi Rural (2012-2014)

vii. East: Kolkata (2012).

Average yearly incident cases were computed by dividing the number of incident cases recorded for each site in each registry by the total number of years, data were recorded for.

Cumulative risk(s) computed by NCRP were based on the following formulae:

Cumulative risk $=100 \times(1-\exp [-$ cumulative rate/100] $)$

Where,

cumulative rate $=(5 \times \Sigma[\mathrm{ASpR}] \times 100) / 100,000$ and

$\mathrm{ASpR}$ is age-specific incidence rate(s)

The multiplication factors five in above formula of cumulative rate indicate the 5-year age intervals in ASpRs.

We computed another indicator, one in a number of persons likely to develop urogenital cancer in the lifetime of 0-64 years, for each urogenital site in each registry as.

One in a number of persons likely to develop urogenital neoplasm $=100 /$ cumulative risk.
Data on indicators, namely number of incident cases, AARs and one in a number of persons develop cancer were summarized for both the sexes in each of the cancer registries and presented region-wise in the form of ranges.

\section{Results}

The proportion of all urogenital neoplasms in comparison to all cancers was $12.51 \%$ in women and $5.93 \%$ in men. The Western region saw the highest number of cases of urogenital neoplasms, both in females (3353) and males (1786) [Table 1]. The highest rate of incidence of urogenital cancers in women was found in the Northeastern region $(23 / 100,000)$, followed by the Southern and Northern regions $(16 / 100,000)$. For men, the highest rate of incidence $(12 / 100,000)$ was noted in the Northern and the Northeastern regions [Table 2]. Risk of development of urogenital cancers for women was maximum (1 in 50) in the Northeastern region. For men, the risk of developing neoplasms of urogenital sites was highest (1 in 250) in the Northern and Northeastern Regions [Table 2]. Overall, the risk of developing urogenital neoplasms (as one in a number of persons likely to develop cancer) ranged from 1 in 50 to 1 in 1000 for women and 1 in 250-1 in 1000 for men [Table 2].

\section{Urologic neoplasms}

Of all urologic neoplasms, bladder neoplasms were associated both with the highest incidence and risk, irrespective of the sexes. It was followed by neoplasms of the kidney. For both the sites, the incidence and the risk were consistently higher for the males as compared to females across all the regions. The incidence of bladder neoplasms in males was the highest in the North $(8 / 100,000)$, the associated risk too was the highest in the North (one in 250). The Northeastern and Northern regions reported the highest incidence of bladder neoplasms $(2 / 100,000)$ for the females, with the risk being the same across all the regions (one in 1000, where applicable) [Table 3].

For males, both the incidence and the risk of developing kidney neoplasms was the highest in the Northern, Southern, and Western Regions (Rate: 3/100,000, Risk: one in 500). The highest incidence of kidney neoplasms was seen in the Western region for females $(2 / 100,000)$. The risk of developing kidney neoplasms in females was same across the regions (one in 1000, where applicable). The Western region had a marginally greater burden of incidence and risk as compared to other regions for both the sexes.

For the neoplasms of the renal pelvis and ureter, both the incidence and risk were quite low for all genders across all the regions. Overall, both the incidences and risk of development of urologic cancers in both the sexes was highest in the North (Rate: 7/100,000; Risk: one in 250) [Table 2]. 
Table 1: Annual Incident Cancer Cases in Females and in Males

\begin{tabular}{|c|c|c|c|c|c|c|c|c|c|c|}
\hline \multicolumn{11}{|c|}{ Annual Incident Cancer Cases in Males } \\
\hline $\begin{array}{l}\text { Region of Registry } \\
\text { location ( } n=\text { No. of } \\
\text { Registries) }\end{array}$ & $\begin{array}{l}\text { Penis } \\
\text { C60 }\end{array}$ & $\begin{array}{c}\text { Prostate } \\
\text { C61 }\end{array}$ & $\begin{array}{l}\text { Testis } \\
\text { C62 }\end{array}$ & $\begin{array}{c}\text { Other male } \\
\text { genital } \\
\text { C63 }\end{array}$ & $\begin{array}{c}\text { Kidney } \\
\text { etc. } \\
\text { C64 }\end{array}$ & $\begin{array}{c}\text { Renal } \\
\text { Pelvis } \\
\text { C65 }\end{array}$ & $\begin{array}{c}\text { Ureter } \\
\text { C66 }\end{array}$ & $\begin{array}{c}\text { Urinary } \\
\text { Bladder } \\
\text { C67 }\end{array}$ & $\begin{array}{c}\text { Unspecified } \\
\text { Urinary Organs } \\
\text { C68 }\end{array}$ & Total \\
\hline North $(n=2)$ & 86 & 744 & 95 & 11 & 210 & 1 & 3 & 485 & 6 & 1641 \\
\hline South $(n=4)$ & 75 & 692 & 52 & 3 & 199 & 1 & 6 & 306 & 2 & 1336 \\
\hline Central $(n=1)$ & 6 & 42 & 11 & 0 & 9 & 1 & 0 & 23 & 0 & 92 \\
\hline Northeast $(n=11)$ & 87 & 157 & 47 & 7 & 82 & 0 & 1 & 142 & 2 & 525 \\
\hline West $(n=7)$ & 159 & 836 & 122 & 6 & 269 & 1 & 4 & 386 & 3 & 1786 \\
\hline Rural West $(n=1)$ & 4 & 7 & 0 & 0 & 1 & 0 & 0 & 4 & 0 & 16 \\
\hline East $(n=1)$ & 25 & 229 & 20 & 0 & 57 & 0 & 0 & 108 & 2 & 441 \\
\hline All regions $(n=27)$ & 442 & 2707 & 347 & 27 & 827 & 4 & 14 & 1454 & 15 & 5837 \\
\hline \multicolumn{7}{|c|}{$\begin{array}{l}\text { Total Annual Cancer Cases (Urogenital Sites) } \\
\text { in all regions }\end{array}$} & 5837 & & & \\
\hline \multicolumn{7}{|c|}{$\begin{array}{l}\text { Total Annual Cancer Cases (All sites) in all } \\
\text { regions }\end{array}$} & 45,645 & & & \\
\hline
\end{tabular}

Table 2: Incidence of Urologic, Genital and Urogenital Neoplasms in different geographical regions of India categorized according to location of population-based cancer registries

\begin{tabular}{|c|c|c|c|c|c|c|c|c|c|}
\hline \multicolumn{10}{|c|}{ AAR (One in number of people likely to develop cancer) } \\
\hline \multirow{2}{*}{$\begin{array}{l}\text { Region of registry } \\
\text { location }(n=\text { No. of } \\
\text { registries) }\end{array}$} & \multicolumn{3}{|c|}{ Urologic Neoplasms (C64-C68) } & \multicolumn{3}{|c|}{$\begin{array}{c}\text { Genital Neoplasms (C50-C58; } \\
\text { C60-C63) }\end{array}$} & \multicolumn{3}{|c|}{$\begin{array}{c}\text { Urogenital Neoplasms (C50-C58; } \\
\text { C60-C63; C64-C68) }\end{array}$} \\
\hline & Female & Male & Both Sexes & Female & Male & Both Sexes & Female & Male & Both Sexes \\
\hline North $(n=2)$ & $\begin{array}{c}0.01-1.53 \\
(1000)\end{array}$ & $\begin{array}{c}0.02-7.41 \\
(250-1000)\end{array}$ & $\begin{array}{c}0.01-7.41 \\
(250-1000)\end{array}$ & $\begin{array}{c}0.08-41 \\
(33-500)\end{array}$ & $\begin{array}{l}0.04-12.39 \\
(333-1000)\end{array}$ & $\begin{array}{c}0.04-41 \\
(33-1000)\end{array}$ & $\begin{array}{c}0.01-41 \\
(33-1000)\end{array}$ & $\begin{array}{l}0.02-12.39 \\
(250-1000)\end{array}$ & $\begin{array}{c}0.01-41 \\
(33-1000)\end{array}$ \\
\hline South $(n=4)$ & $\begin{array}{l}0.09-1.11 \\
(1000)\end{array}$ & $\begin{array}{c}0.03-4.9 \\
(500-1000)\end{array}$ & $\begin{array}{c}0.03-4.9 \\
(500-1000)\end{array}$ & $\begin{array}{c}0.02-37.92 \\
(34-1000)\end{array}$ & $\begin{array}{c}0.05-9.4 \\
(500-1000)\end{array}$ & $\begin{array}{c}0.02-37.92 \\
(34-1000)\end{array}$ & $\begin{array}{r}0.02-37.93 \\
(34-1000)\end{array}$ & $\begin{array}{c}0.03-9.4 \\
(500-1000)\end{array}$ & $\begin{array}{c}0.02-37.93 \\
(34-1000)\end{array}$ \\
\hline Central $(n=1)$ & $\begin{array}{c}0.04-0.72 \\
(-)\end{array}$ & $\begin{array}{l}0.11-2.95 \\
(1000)\end{array}$ & $\begin{array}{l}0.04-2.95 \\
(1000)\end{array}$ & $\begin{array}{l}0.06-33.05 \\
(40-500)\end{array}$ & $\begin{array}{c}0.64-5.59 \\
(500-1000)\end{array}$ & $\begin{array}{r}0.06-33.05 \\
(40-1000)\end{array}$ & $\begin{array}{c}0.04-33.05 \\
(40-500)\end{array}$ & $\begin{array}{c}0.11-5.59 \\
(500-1000)\end{array}$ & $\begin{array}{r}0.04-33.05 \\
(40-1000)\end{array}$ \\
\hline Northeast $(n=11)$ & $\begin{array}{l}0.02-1.91 \\
(1000)\end{array}$ & $\begin{array}{c}0.03-3.78 \\
(500-1000)\end{array}$ & $\begin{array}{c}0.02-3.78 \\
(500-1000)\end{array}$ & $\begin{array}{l}0.03-27.11 \\
(50-1000)\end{array}$ & $\begin{array}{l}0.12-12.16 \\
(250-1000)\end{array}$ & $\begin{array}{c}0.03-27.11 \\
(50-1000)\end{array}$ & $\begin{array}{r}0.02-27.11 \\
(50-1000)\end{array}$ & $\begin{array}{l}0.03-12.16 \\
(250-1000)\end{array}$ & $\begin{array}{r}0.02-27.11 \\
(50-1000)\end{array}$ \\
\hline West $(n=7)$ & $\begin{array}{c}0.02 \\
(1000)\end{array}$ & $\begin{array}{c}0.01-4.06 \\
(500-1000)\end{array}$ & $\begin{array}{l}0.01-4.06 \\
(500-1000)\end{array}$ & $\begin{array}{c}0.04-33.63 \\
(43-1000)\end{array}$ & $\begin{array}{c}0.06-9.78 \\
(500-1000)\end{array}$ & $\begin{array}{c}0.04-33.63 \\
(43-1000)\end{array}$ & $\begin{array}{c}0.02-33.63 \\
(43-1000)\end{array}$ & $\begin{array}{c}0.01-9.78 \\
(500-1000)\end{array}$ & $\begin{array}{c}0.01-33.63 \\
(43-1000)\end{array}$ \\
\hline Rural West $(n=1)$ & $\begin{array}{l}0.2-0.81 \\
(1000)\end{array}$ & $\begin{array}{l}0.39-1.57 \\
(1000)\end{array}$ & $\begin{array}{c}0.2-1.57 \\
(1000)\end{array}$ & $\begin{array}{l}0.13-16.09 \\
(83-1000)\end{array}$ & $\begin{array}{l}0.12-2.11 \\
(1000)\end{array}$ & $\begin{array}{c}0.12-16.09 \\
(83-1000)\end{array}$ & $\begin{array}{c}0.13-16.09 \\
(83-1000)\end{array}$ & $\begin{array}{l}0.12-2.11 \\
(1000)\end{array}$ & $\begin{array}{r}0.12-16.09 \\
(83-1000)\end{array}$ \\
\hline East $(n=1)$ & $\begin{array}{c}0.05-1.08 \\
(1000)\end{array}$ & $\begin{array}{c}0.13-3.96 \\
(1000)\end{array}$ & $\begin{array}{c}0.05-3.96 \\
(1000)\end{array}$ & $\begin{array}{c}0.03-25.52 \\
(53-1000)\end{array}$ & $\begin{array}{c}0.68-8.21 \\
(1000)\end{array}$ & $\begin{array}{c}0.03-25.52 \\
(53-1000)\end{array}$ & $\begin{array}{c}0.03-25.52 \\
(4-3333)\end{array}$ & $\begin{array}{c}0.13-8.21 \\
(1000)\end{array}$ & $\begin{array}{c}0.03-25.52 \\
(53-1000)\end{array}$ \\
\hline All regions $(n=27)$ & $\begin{array}{c}0.01-1.91 \\
(1000)\end{array}$ & $\begin{array}{c}0.11-7.41 \\
(250-1000)\end{array}$ & $\begin{array}{c}0.01-7.41 \\
(250-1000)\end{array}$ & $\begin{array}{c}0.02-41 \\
(33-1000)\end{array}$ & $\begin{array}{l}0.04-12.39 \\
(250-1000)\end{array}$ & $\begin{array}{c}0.02-41 \\
(33-1000)\end{array}$ & $\begin{array}{c}0.01-41 \\
(4-3333)\end{array}$ & $\begin{array}{l}0.01-12.39 \\
(250-1000)\end{array}$ & $\begin{array}{c}0.01-41 \\
(33-1000)\end{array}$ \\
\hline
\end{tabular}

Geographical distribution of cancer registries: North - Delhi, Patiala District; South - Bengaluru, Chennai, Kollam, Thiruvananthapuram; Central - Bhopal; Northeast - Cachar District, Kamrup Urban District, Dibrugarh, Manipur State, Mizoram State, Sikkim State,

Meghalaya, Tripura State, Nagaland, Naharlagun District, Pasighat; West - Barshi Expanded, Mumbai, Ahmedabad Urban, Aurangabad,

Nagpur, Pune, Wardha District; Rural West - Barshi Rural; East - Kolkata

\section{Genital neoplasms (female)}

Northeast saw the highest incident burden of cervical neoplasms $(23 / 100,000)$ followed by South, North, and Rural west $(16 / 100,000)$. Risk of developing cervical neoplasms followed along; one in 50 in the Northeast and one in 83 in the South, North, and Rural West [Table 4]. The incidence and the risk of developing neoplasms of uterine body were highest in the Southern and Northern regions $(6 / 100,000$; one in 250), followed closely by Rural West and East (4/100,000; one in 333). For incident ovarian neoplasms, the Northern region came at the top $(10 / 100,000)$, followed closely by Northeast $(9 / 100,000)$. Both regions displayed the highest risk (one in 143) compared to other regions. While the incidence of vaginal neoplasm was low in all the regions, the incidences in the West, Rural West, and South were slightly higher $(2 / 100,000)$. The incidences of 


\begin{tabular}{|c|c|c|c|c|c|c|c|c|c|c|}
\hline \multirow[b]{3}{*}{$\begin{array}{l}\text { Region of } \\
\text { registry } \\
\text { location ( } n=\text { No. } \\
\text { of Registries) } \\
\end{array}$} & \multicolumn{10}{|c|}{ AAR (One in number of people likely to develop cancer) } \\
\hline & \multicolumn{5}{|c|}{ Female } & \multicolumn{5}{|c|}{ Male } \\
\hline & $\begin{array}{l}\text { Kidney } \\
\text { etc. } \\
\text { C64 }\end{array}$ & $\begin{array}{l}\text { Renal } \\
\text { Pelvis } \\
\text { C65 }\end{array}$ & $\begin{array}{l}\text { Ureter } \\
\text { C66 }\end{array}$ & $\begin{array}{c}\text { Urinary } \\
\text { Bladder } \\
\text { C67 }\end{array}$ & $\begin{array}{c}\text { Unspecified } \\
\text { Urinary Organs } \\
\text { C68 }\end{array}$ & $\begin{array}{l}\text { Kidney } \\
\text { etc. } \\
\text { C64 }\end{array}$ & $\begin{array}{l}\text { Renal } \\
\text { Pelvis } \\
\text { C65 }\end{array}$ & $\begin{array}{l}\text { Ureter } \\
\text { C66 }\end{array}$ & $\begin{array}{l}\text { Urinary } \\
\text { Bladder } \\
\text { C67 }\end{array}$ & $\begin{array}{c}\text { Unspecified } \\
\text { Urinary Organs } \\
\text { C68 }\end{array}$ \\
\hline North $(n=2)$ & $\begin{array}{c}0.98-1.3 \\
(1000)\end{array}$ & $0.01(-)$ & $-(-)$ & $\begin{array}{c}1.05-1.53 \\
(1000)\end{array}$ & $0.03-0.03(-)$ & $\begin{array}{c}1.64-2.85 \\
(500-1000)\end{array}$ & $\begin{array}{c}0.02 \\
(-)\end{array}$ & $\begin{array}{c}0.05 \\
(-)\end{array}$ & $\begin{array}{c}3.6-7.41 \\
(250-500)\end{array}$ & $0.06(-)$ \\
\hline South $(n=4)$ & $\begin{array}{c}0.69-1.11 \\
(1000)\end{array}$ & $0.01(-)$ & $\begin{array}{c}0.03-0.07 \\
(-)\end{array}$ & $\begin{array}{c}0.52-1.09 \\
(1000)\end{array}$ & $0.02-0.04(-)$ & $\begin{array}{c}1.9-2.76 \\
(500-1000)\end{array}$ & $\begin{array}{c}0.03 \\
(-)\end{array}$ & $\begin{array}{c}0.13 \\
(-)\end{array}$ & $\begin{array}{c}2.39-4.9 \\
(500-1000)\end{array}$ & $0.07(-)$ \\
\hline Central $(n=1)$ & $0.61(-)$ & $0.04(-)$ & $-(-)$ & $0.72(-)$ & $-(-)$ & $\begin{array}{c}1.03 \\
(1000)\end{array}$ & $\begin{array}{c}0.11 \\
(-)\end{array}$ & $-(-)$ & $\begin{array}{c}2.95 \\
(1000)\end{array}$ & $-(-)$ \\
\hline $\begin{array}{l}\text { Northeast } \\
(n=11)\end{array}$ & $\begin{array}{c}0.19-1.04 \\
(-)\end{array}$ & $\begin{array}{c}0.02-0.04 \\
(-)\end{array}$ & $\begin{array}{c}0.07-0.3 \\
(-)\end{array}$ & $\begin{array}{c}0.11-1.91 \\
(1000)\end{array}$ & $0.03-0.05(-)$ & $\begin{array}{c}0.13-2.3 \\
(1000)\end{array}$ & $\begin{array}{c}0.03 \\
(-)\end{array}$ & $\begin{array}{c}0.15 \\
(-)\end{array}$ & $\begin{array}{c}0.29-3.78 \\
(500)\end{array}$ & $0.25(-)$ \\
\hline West $(n=7)$ & $\begin{array}{c}0.35-1.52 \\
(1000)\end{array}$ & $\begin{array}{c}0.03-0.04 \\
(-)\end{array}$ & $0.02(-)$ & $\begin{array}{l}0.38-1 \\
(1000)\end{array}$ & $0.02-0.02(-)$ & $\begin{array}{c}0.54-2.71 \\
(500)\end{array}$ & $\begin{array}{c}0.01 \\
(-)\end{array}$ & $\begin{array}{c}0.05 \\
(-)\end{array}$ & $\begin{array}{c}0.83-4.06 \\
(1000)\end{array}$ & $0.04(-)$ \\
\hline $\begin{array}{l}\text { Rural West } \\
(n=1)\end{array}$ & $0.2(-)$ & $-(-)$ & $-(-)$ & $\begin{array}{c}0.81 \\
(1000)\end{array}$ & $-(-)$ & $0.39(-)$ & $-(-)$ & $-(-)$ & $\begin{array}{c}1.57 \\
(1000)\end{array}$ & $-(-)$ \\
\hline East $(n=1)$ & $\begin{array}{c}1.08 \\
(1000)\end{array}$ & $-(-)$ & $0.05(-)$ & $0.77(-)$ & $0.12(-)$ & $2.02(-)$ & $-(-)$ & $-(-)$ & $\begin{array}{c}3.96 \\
(1000)\end{array}$ & $0.13(-)$ \\
\hline $\begin{array}{l}\text { All regions } \\
(n=27)\end{array}$ & $\begin{array}{c}0.19-1.52 \\
(1000)\end{array}$ & $\begin{array}{c}0.01-0.04 \\
(-)\end{array}$ & $\begin{array}{c}0.3-0.7 \\
(-)\end{array}$ & $\begin{array}{c}0.11-1.91 \\
(1000)\end{array}$ & $0.02-0.12(-)$ & $\begin{array}{c}0.13-2.85 \\
(500-1000)\end{array}$ & $\begin{array}{c}0.11 \\
(-)\end{array}$ & $\begin{array}{c}0.15 \\
(-)\end{array}$ & $\begin{array}{c}0.29-7.41 \\
(250-1000)\end{array}$ & $0.25(-)$ \\
\hline
\end{tabular}

Geographical distribution of cancer registries: North - Delhi, Patiala District; South - Bengaluru, Chennai, Kollam, Thiruvananthapuram; Central - Bhopal; Northeast - Cachar District, Kamrup Urban District, Dibrugarh, Manipur State, Mizoram State, Sikkim State, Meghalaya, Tripura State, Nagaland, Naharlagun District, Pasighat; West - Barshi Expanded, Mumbai, Ahmedabad Urban, Aurangabad, Nagpur, Pune, Wardha District; Rural West - Barshi Rural; East - Kolkata

Table 4: Incidence of Female Genital Neoplasms in different geographical regions of India categorized according to location of population-based cancer registries

\begin{tabular}{|c|c|c|c|c|c|c|c|c|}
\hline \multicolumn{9}{|c|}{ AAR (One in number of people likely to develop cancer) } \\
\hline $\begin{array}{l}\text { Region of registry } \\
\text { location }(n=\text { No. of }\end{array}$ & Vulva & Vagina & $\begin{array}{l}\text { Cervix } \\
\text { Uteri }\end{array}$ & $\begin{array}{c}\text { Corpus } \\
\text { Uteri }\end{array}$ & $\begin{array}{c}\text { Uterus } \\
\text { Unspecified }\end{array}$ & Ovary etc. & $\begin{array}{c}\text { Other Female } \\
\text { Genital }\end{array}$ & Placenta \\
\hline Registries) & $\mathbf{C 5 1}$ & $\mathbf{C 5 2}$ & C53 & C54 & C55 & C56 & C57 & C58 \\
\hline North $(n=2)$ & $\begin{array}{c}0.43-0.6 \\
(-)\end{array}$ & $\begin{array}{c}0.46-0.52 \\
(-)\end{array}$ & $\begin{array}{c}11.46-15.53 \\
(83-100)\end{array}$ & $\begin{array}{l}3.16-5.52 \\
(250-333)\end{array}$ & $\begin{array}{c}0.46-3.03 \\
(500)\end{array}$ & $\begin{array}{l}5.52-10.02 \\
(143-250)\end{array}$ & $0.09-0.09(-)$ & $\begin{array}{c}0.05-0.08 \\
(-)\end{array}$ \\
\hline South $(n=4)$ & $\begin{array}{c}0.05-0.57 \\
(-)\end{array}$ & $\begin{array}{c}0.18-0.79 \\
(1000)\end{array}$ & $\begin{array}{c}6.69-15.88 \\
(83-200)\end{array}$ & $\begin{array}{l}2.92-6.04 \\
(250-500)\end{array}$ & $\begin{array}{c}0.22-1.19 \\
(1000)\end{array}$ & $\begin{array}{c}5.42-8.2 \\
(167-250)\end{array}$ & $0.02-0.1(-)$ & $\begin{array}{c}0.04-0.08 \\
(-)\end{array}$ \\
\hline Central $(n=1)$ & $0.26(-)$ & $0.59(-)$ & $13.83(100)$ & $2.77(500)$ & $0.06(-)$ & $8.4(200)$ & $-(-)$ & $0.41(-)$ \\
\hline Northeast $(n=11)$ & $\begin{array}{c}0.2-0.86 \\
(1000)\end{array}$ & $\begin{array}{l}0.08-1 \\
(1000)\end{array}$ & $\begin{array}{c}4.91-23.07 \\
(50-250)\end{array}$ & $\begin{array}{c}0.15-3.93 \\
(333-1000)\end{array}$ & $\begin{array}{c}0.38-2.09 \\
(500-1000)\end{array}$ & $\begin{array}{l}1.72-8.68 \\
(143-500)\end{array}$ & $0.03-0.2(-)$ & $\begin{array}{c}0.06-0.42 \\
(-)\end{array}$ \\
\hline West $(n=7)$ & $\begin{array}{c}0.08-0.43 \\
(-)\end{array}$ & $\begin{array}{c}0.12-1.29 \\
(1000)\end{array}$ & $\begin{array}{c}6.91-14.65 \\
(91-200)\end{array}$ & $\begin{array}{c}0.9-4.17 \\
(333-1000)\end{array}$ & $\begin{array}{c}0.06-0.88 \\
(1000)\end{array}$ & $\begin{array}{l}2.32-8.14 \\
(167-500)\end{array}$ & $0.04-0.15(-)$ & $\begin{array}{c}0.04-0.09 \\
(-)\end{array}$ \\
\hline Rural West $(n=1)$ & $0.2(-)$ & $\begin{array}{c}0.97 \\
(1000)\end{array}$ & $16.09(83)$ & $\begin{array}{c}0.88 \\
(1000)\end{array}$ & $0.13(-)$ & $2.93(500)$ & $-(-)$ & $-(-)$ \\
\hline East $(n=1)$ & $0.49(-)$ & $0.54(-)$ & $10.43(125)$ & $3.94(333)$ & $1.16(1000)$ & $7.96(167)$ & $0.03(-)$ & $-(-)$ \\
\hline All regions $(n=27)$ & $\begin{array}{c}0.05-0.86 \\
(1000)\end{array}$ & $\begin{array}{c}0.08-1.29 \\
(1000)\end{array}$ & $\begin{array}{c}4.91-23.07 \\
(50-250)\end{array}$ & $\begin{array}{c}0.15-6.04 \\
(250-1000)\end{array}$ & $\begin{array}{c}0.06-3.03 \\
(500-1000)\end{array}$ & $\begin{array}{l}1.72-10.02 \\
(143-500)\end{array}$ & $0.02-0.2(-)$ & $\begin{array}{c}0.04-0.42 \\
(-)\end{array}$ \\
\hline
\end{tabular}

Geographical distribution of cancer registries: North - Delhi, Patiala District; South - Bengaluru, Chennai, Kollam, Thiruvananthapuram; Central - Bhopal; Northeast - Cachar District, Kamrup Urban District, Dibrugarh, Manipur State, Mizoram State, Sikkim State, Meghalaya, Tripura State, Nagaland, Naharlagun District, Pasighat; West - Barshi Expanded, Mumbai, Ahmedabad Urban, Aurangabad, Nagpur, Pune, Wardha District; Rural West - Barshi Rural; East - Kolkata

vulvar, placental, and other female neoplasms were quite low. Cervical neoplasms had the highest incidence among female genital neoplasms, followed by neoplasms of uterine body. The Northeastern region showed the highest incident burden and risk of female genital neoplasms, followed by the Rural West, South, and North [Table 2]. 


\begin{tabular}{|c|c|c|c|c|}
\hline \multicolumn{5}{|c|}{ AAR (One in number of people likely to develop cancer) } \\
\hline Region of registry location & Penis & Prostate & Testis & Other Male Genital \\
\hline$(n=$ No. of registries $)$ & $\mathbf{C 6 0}$ & C61 & C62 & $\mathrm{C63}$ \\
\hline North $(n=2)$ & $1.03-1.12(1000)$ & $6.3-12.39(333-500)$ & $0.64-0.85(1000)$ & $0.04-0.14(-)$ \\
\hline South $(n=4)$ & $0.45-1.16(1000)$ & $6.14-9.4(500-1000)$ & $0.33-0.75(1000)$ & $0.05(-)$ \\
\hline Central $(n=1)$ & $0.64(-)$ & $5.59(500)$ & $0.95(1000)$ & $-(-)$ \\
\hline Northeast $(n=11)$ & $0.69-1.66(1000)$ & $0.82-12.16(250)$ & $0.12-1.29(1000)$ & $0.2(-)$ \\
\hline West $(n=7)$ & $0.62-1.46(1000)$ & $1.48-9.78(500)$ & $0.34-0.81(-)$ & $0.06(-)$ \\
\hline Rural West $(n=1)$ & $1.68(1000)$ & $2.11(1000)$ & $0.12(-)$ & $-(-)$ \\
\hline East $(n=1)$ & $0.88(1000)$ & $8.21(1000)$ & $0.68(-)$ & $-(-)$ \\
\hline All regions $(n=27)$ & $0.45-1.68(1000)$ & $0.82-12.39(250-1000)$ & $0.12-1.29(1000)$ & $0.04-0.2(-)$ \\
\hline
\end{tabular}

Geographical distribution of cancer registries: North - Delhi, Patiala District; South - Bengaluru, Chennai, Kollam, Thiruvananthapuram; Central - Bhopal; Northeast - Cachar District, Kamrup Urban District, Dibrugarh, Manipur State, Mizoram State, Sikkim State, Meghalaya, Tripura State, Nagaland, Naharlagun District, Pasighat; West - Barshi Expanded, Mumbai, Ahmedabad Urban, Aurangabad, Nagpur, Pune, Wardha District; Rural West - Barshi Rural; East - Kolkata

\section{Genital neoplasms (male)}

Among male genital cancers, the highest rate of incidence $(12 / 100,000)$ and risk (one in 250) was for prostate cancer. The Northeastern and Northern regions showed the highest incidence. The highest risk was noted in the Northeastern region, followed by Northern region (one in 333). The incidence of penile neoplasms was the highest in the Rural West $(2 / 100,000)$. The risk of development was similar across all the regions (one in 1000; where applicable) [Table 5]. Other male genital sites showed appreciably lower incidence. Overall, men in the Northern and Northeastern regions had the highest incidence and risk of development of neoplasms of the male genital tract [Table 2].

\section{Discussion}

Gender differences were observed for the incidence and risk profiles of specific and generalized sites. Both the incidence and the risk of developing urologic neoplasms were almost four times higher in males as compared to females across all the regions. The higher risk of urologic neoplasm in males is due to higher occupational exposure and smoking among males. ${ }^{[8-10]}$ The incidence of neoplasms of the female genital tract was more than three times the incidence of neoplasms of the male genital tract. For both the genders, the genital tract neoplasms contributed a greater proportion to the overall urogenital neoplasm incidence and risk. This was, especially true for the female gender, which suffered from lower incidence and risk of urologic neoplasms, and very high incidence and risk of genital tract neoplasms. The complex interplay between endocrinal and reproductive factors in the maintenance of fertile female genitalia has been implicated.

Cervical neoplasms were the most common site of female genital neoplasms. Incidence and risk of development of cervical cancer have increased compared to GLOBOCAN
2012 report and from an earlier study utilizing 2007-2008 data. ${ }^{[1,11]}$ The northeastern region has seen a substantial increase in risk (from 1 in 82 to 1 in 50) since then. HPV infection is the most important factor significantly associated with the development of cervical cancer. Lack of a national screening program and high costs of HPV vaccination, ${ }^{[3,12,13]}$ stand as a major roadblock in improving outcomes associated with cervical cancer. The incidence of uterine neoplasms has increased almost two-fold compared to values reported by a study utilizing data spanning 1980-2003 from a limited set of registries. ${ }^{[14]}$ Compared to the same study, incidence of ovarian neoplasms too showed an increase albeit of lesser magnitude.

Prostate was the most common site of male genital neoplasms. Despite conventionally low incidences in Asian and African countries, earlier studies had indicated a slowly but steadily rising burden. ${ }^{[15,16]}$ The values reported by present study conform to this trend. The urinary bladder was the most common site for the development of urologic neoplasms, followed by kidney. Smoking is an established risk factor for both the sites. ${ }^{[17,18]}$ In addition, occupational exposure is an independent risk factor for the development of bladder neoplasms. ${ }^{[8]}$

The site-specific neoplasms contributing to the bulk of urogenital neoplasm burden, all have risk factors that can be directly addressed. By reducing smoking, HPV prevalence, and occupational exposure, the burden of urogenital neoplasms can be substantially reduced. What lacking is the incorporation of risk profile assessment and dissemination of advice at appropriate centers.

\section{Conclusions}

Making people aware of urogenital neoplasms and their risk factors are important for the public health awareness point of view. Centers that deal with either management of urogenital cases or/and screening of genital neoplasms 
could serve as the designated centers for creating such awareness. Efforts can be concentrated in regions with higher incidence/risk, namely the Northern and Southern regions. In addition, there is a dire need to increase the number of Population-Based Cancer Registries (PBCRs) across the regions to allow for a more wholesome visualization of distribution.

\section{Financial support and sponsorship}

Nil.

\section{Conflicts of interest}

There are no conflicts of interest.

\section{References}

1. Ferlay J, Soerjomataram I, Ervik M, Dikshit R, Eser S, Mathers C, et al. GLOBOCAN 2012 v1.0, Cancer Incidence and Mortality Worldwide: IARC Cancer Base No 11. Lyon, France: International Agency for Research on Cancer; 2013. Available from: http://www.globocan.iarc.fr. [Last accessed on 2017 Mar 03].

2. Mattheij I, Pollock AM, Brhlikova P. Do cervical cancer data justify HPV vaccination in India? Epidemiological data sources and comprehensiveness. J R Soc Med 2012;105:250-62.

3. Krishnan S, Madsen E, Porterfield D, Varghese B. Advancing cervical cancer prevention in India: Implementation science priorities. Oncologist 2013;18:1285-97.

4. Chawla PC, Chawla AK, Shrivastava R, Shrivastava A, Chaudhary S. Situation analysis of existing facilities for screening, treatment and prevention of cervical cancer in hospitals/primary health centers of Delhi-NCR region, India. Asian Pac J Cancer Prev 2014;15:5475-82.

5. Raj S, Piang LK, Nair KS, Tiwari VK, Kaur H, Singh B, et al. Awareness regarding risk factors, symptoms and treatment facilities for cancer in selected states of India. Asian Pac J Cancer Prev 2012;13:4057-62.

6. Rashid S, Labani S, Das BC. Knowledge, awareness and attitude on HPV, HPV vaccine and cervical cancer among the college students in India. PLoS One 2016;11:e0166713.

7. Wadhwa P. The current three-year postgraduate program in urology is insufficient to train urologists: Against the motion. Indian J Urol 2008;24:339-42.

8. Letašiová S, Medve'ová A, Šovčíková A, Dušinská M, Volkovová K, Mosoiu C, et al. Bladder cancer, a review of the environmental risk factors. Environ Health 2012;11 Suppl 1:S11.

9. Hemelt M, Yamamoto H, Cheng KK, Zeegers MP. The effect of smoking on the male excess of bladder cancer: A meta-analysis and geographical analyses. Int J Cancer 2009;124:412-9.

10. Global Adult Tobacco Survey (GATS) India: 2009-2010. Mumbai: IIPS, Ministry of Health and Family Welfare, GOI; 2010. Available from: http://www.searo.who.int/LinkFiles/ Regional_Tobacco_Surveillance_System_GATS_India.pdf. [Last accessed on 2017 Mar 29].

11. Asthana S, Chauhan S, Labani S. Breast and cervical cancer risk in India: An update. Indian J Public Health 2014;58:5-10.

12. Sankaranarayanan R. Screening for cancer in low- and middle-income countries. Ann Glob Health 2014;80:412-7.

13. Mishra GA, Pimple SA, Shastri SS. Prevention of cervix cancer in India. Oncology 2016;91 Suppl 1:1-7.

14. Yeole BB. Trends in cancer incidence in female breast, cervix uteri, corpus uteri, and ovary in India. Asian Pac J Cancer Prev 2008;9:119-22.

15. Jain S, Saxena S, Kumar A. Epidemiology of prostate cancer in India. Meta Gene 2014;2:596-605.

16. Lalitha K, Suman G, Pruthvish S, Mathew A, Murthy NS. Estimation of time trends of incidence of prostate cancer - An Indian scenario. Asian Pac J Cancer Prev 2012; $13: 6245-50$.

17. WHO Document. Tobacco smoking. IARC Monographs on the Evaluation of the carcinogenic Risk of Chemicals to Humans. Vol. 38. Lyon: IARC; 1986. Available from: http://monographs. iarc.fr/ENG/Monographs/vol1-42/mono38.pdf. [Last accessed on 2017 Mar 03].

18. Hunt JD, van der Hel OL, McMillan GP, Boffetta P, Brennan P. Renal cell carcinoma in relation to cigarette smoking: Meta-analysis of 24 studies. Int J Cancer 2005;114:101-8. 